\section{Surgical resection of neoplastic cervical spine lesions in relation to the vertebral artery V2 segment}

\author{
Mohamed Al Barbarawi,' Ziad Odat,' \\ Mwaffaq Alheis, ${ }^{3}$ Suhair Qudsieh, ${ }^{4}$ \\ Tareq Qudsieh' \\ 'Department of Neurosurgery, \\ ${ }^{2}$ Department of Orthopedics, and \\ ${ }^{3}$ Department of Radiology, King Abdullah \\ University Hospital, Jordan University of \\ Science and Technology, Irbid, Jordan; \\ ${ }^{4}$ Hashemite University, Faculty of \\ Medicine, Zarqa, Jordan
}

\section{Abstract}

Neoplastic cervical spine lesions are seen infrequently by the spinal surgeon. The surgical management of these tumors, particularly with associated neurovascular compromise, is challenging in terms of achieving proper resection and spinal stabilization and ensuring no subsequent recurrence or failure of fixation. In this report we highlight some of the problems encountered in the surgical management of tumors involving the cervical spine with techniques applied for gross total resection of the tumor without compromising the vertebral arteries. Ten patients with neoplastic cervical spine lesions were managed in our study. The common cardinal presentation was neck and arm pain with progressive cervical radiculo-myelopathy. All patients had plain Xrays, computer tomography scans, and magnetic resonance imaging of the cervical spine. Digital subtraction or magnetic resonance angiograms were performed on both vertebral arteries when the pathology was found to be in proximity to the vertebral artery. When a tumor blush with feeders was evident, endovascular embolization to minimize intraoperative bleeding was also considered. A single approach or a combined anterior cervical approach for corpectomy and cage-with-plate fixation and posterior decompression for resection of the rest of the tumor with spinal fixation was then accomplished as indicated. All cases made a good neurological recovery and had no neural or vascular complications. On the long-term follow-up of the survivors there was no local recurrence or surgical failure. Only three patients died: two from the primary malignancy and one from pulmonary embolism. This report documents a safe and reliable way to deal with neoplastic cervical spine lesions in proximity to vertebral arteries with preservation of both arteries.

\section{Introduction}

Non-traumatic cervical spine lesions are frequent and include neoplastic, spondylotic, infectious, and inflammatory lesions, or congenital anomalies. These lesions may be seen in any age group and affect both males and females equally.,2 Symptoms and signs are variable and extend from a localized or radicular pain to tetraplegia. Progressive and gradual cervical myelopathy is common but fleeting symptoms may also be present. ${ }^{3}$ Tumor extension to the adjacent structures including bone, neural, and vertebral artery (VA), and the mobility of the spine raises technical complexities in the surgical approach. The surgical technique includes resection of the tumor and stabilization of the spine. ${ }^{4}$ Intraoperative identification of the VA (V2 segment ) is necessary when a meticulous step-by-step surgical technique is affected; the course of the V2 segment is in and between the transverse processes from vertebrae $\mathrm{C} 6$ to $\mathrm{C} 2$. It usually passes medially to the sternocleidomastoid muscle and laterally to the internal jugular vein. This segment is protected by the transverse processes even with pathologies displacing the VA along its course. Reaching the transverse process is the best way to identify the artery. The V2 segment can be exposed by opening the transverse process in the subperiosteal plane, where this portion of the artery is surrounded by a venous plexus and a periosteal sheath. This sheath provides safety to the VA dissection. ${ }^{5}$

In this report we analyzed ten cases who suffered neoplastic cervical spine lesions very close to the V2 segment of the VA, causing cervical radiculo-myelopathy. The surgical options applied for gross total resection and preservation of the VA alongside the neural components are discussed.

\section{Materials and Methods}

The study was approved by the ethical committee for human research (IRB) at Jordan University of Science and Technology. The study group was treated for cervical spine tumors at King Abdullah University Hospital between 2004 and 2009. The diagnosis of cervical spine tumors was based on the clinical presentation, computer tomography (CT) scans, and magnetic resonance imaging (MRI) findings, and on the final histopathology report. Exclusion criteria included patients with spondylotic cervical myelopathy, acute cervical spinal trauma, and intramedullary spinal cord lesions. The study group consisted of six males and four male patients with a median age of 28 years (range: 18-65 years).
Correspondence: Mohamed Al Barbarawi, Division of Neurosurgery, Department of Neuroscience, Faculty of Medicine, Jordan University of Science and Technology, Irbid, Jordan. E-mail: dr_barbarawi@yahoo.com

Key words: cervical spine tumors, spinal fixation, vertebral artery, radical resection, vascular complications.

Received for publication: 23 February 2010.

Revision received: 16 April 2010.

Accepted for publication: 5 May 2010.

This work is licensed under a Creative Commons Attribution 3.0 License (by-nc 3.0).

(C) Copyright Al Barbarawi M. et al., 2010

Licensee PAGEPress, Italy

Neurology International 2010; 2:e11

doi:10.4081/ni.2010.e11

Duration of symptoms to treatment ranged between 1 month and 1 year. The long-term follow-up period extended from 2 to 48 months with an average of 2.8 years.

Two patients had a craniocervical junction meningioma; these lesions distorting the VA, spinal cord, and adjacent nerve roots. One patient had a giant cell tumor that caused an osteolytic lesion and engulfed the VA (Figure 1). There was a 38-week pregnant woman with a vertebral body cavernous hemangioma and exophytic extension causing spinal cord compression and acute paralysis. One case had a cystic odontoid lesion with $\mathrm{C} 2 / 3$ subluxation (Figure 2). The histopathology revealed an osteoblastoma. Another three patients had neurofibromatosis: two of them had a C6 neurofibroma with a dumbbell appearance and the VA was intimately related to the lesion; the third patient had a large suboccipital C2 neurofibroma, which was very close to the VA. There were two patients who had cervical spine malignancy: one of them had metastasis from renal cell carcinoma to the cervical spine, and the other one had a malignant $\mathrm{C} 2$ nerve root sheath tumor; these malignant tumors involved the vertebral elements and caused bony destruction with neural compression and VA involvement.

Neck and arm pain with progressive weakness, spastic gait, and subsequent sphincteric problems were the common cardinal presenting symptoms. Preoperative work-up included a complete blood count, and inflammatory markers such as erythrocyte sedimentation rate, C-reactive protein, and PPD to rule out infectious processes in certain cases. Plain Xrays, CT scans, and MRI of the cervical spine with contrast were done routinely. Malignancy work-up included chest and abdomen-pelvic CT scans, bone scans, a mammogram in 


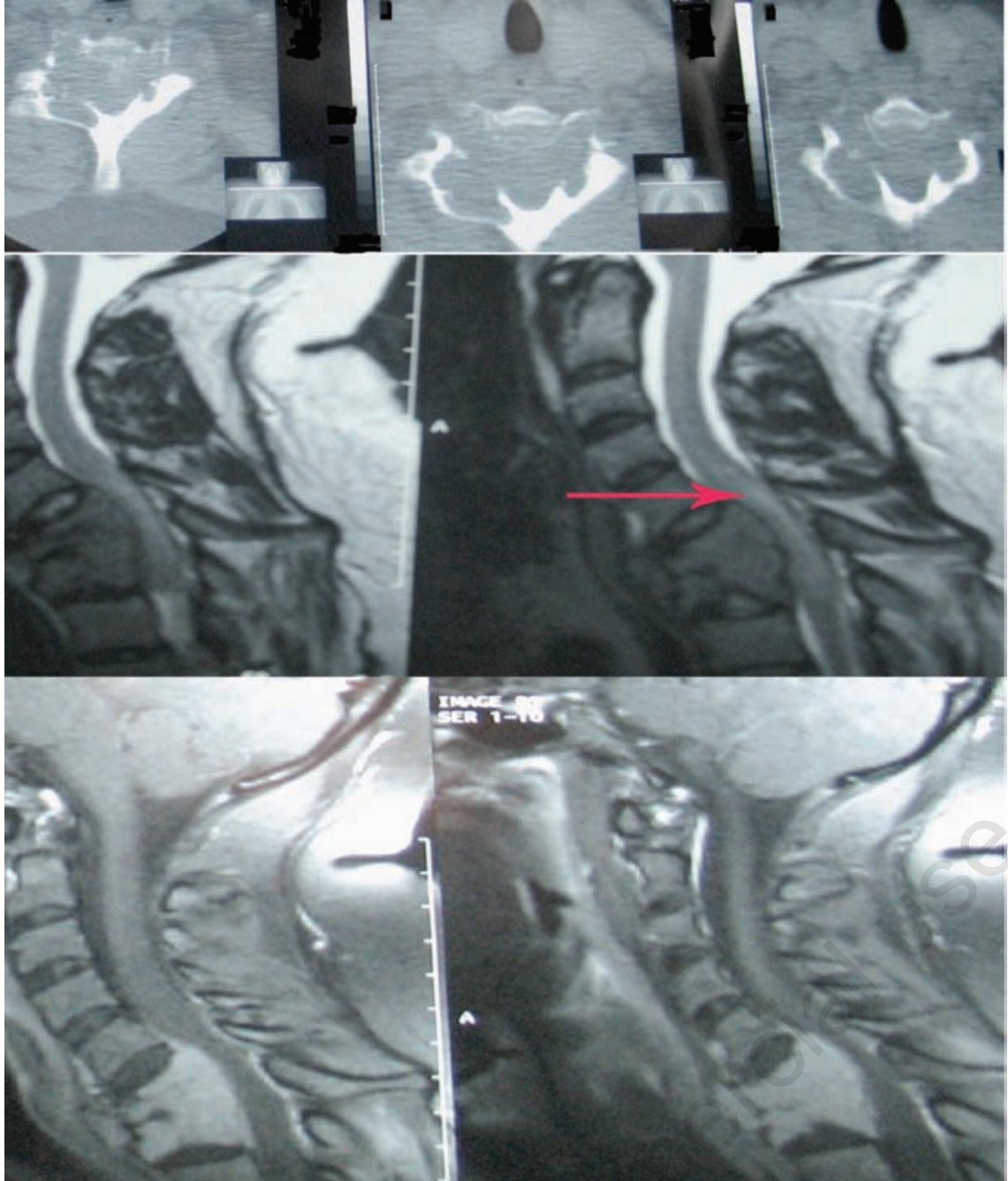

Figure 1. Computer tomography scans showing axial slices of the cervical spine at C6 level (upper three illustrations). T2WI and T1WI post-contrast sagittal magnetic resonance images (lower four illustrations) reveal a C6, C7 vertebral body destructive lesion with kyphotic angulation of the lower cervical spine and retropulsion of the posterior elements of the vertebral body, causing a significant spinal cord compression and buckling (red arrow). The lesion extends into the nerve root exit foramina and to the prevertebral space. A spinal cord high signal is also noted.

females in cases considered suspicious, and a fine needle aspiration performed on cases of suspicious malignancy. Either a digital subtraction or a magnetic resonance angiogram as a road map for the VAs was done in cases with extensive and enhancing lesions on the MRI. Endovascular embolization of the tumor feeders was carried out to the highly vascular lesions a day before the planned surgery to minimize intraoperative profuse bleeding (Figure 3A and B).

Anterior or posterior surgical approaches with or without spinal fixation were considered as the primary surgical technique utilized to achieve a gross total resection of the lesion. However, staged approaches were planned to deal with destructive and extensive lesions on two cases, treated initially with an anterior approach for corpectomy and fixation with cage and plates. A posterior approach for completion of the tumor resection where feasible and spinal fixation were accomplished on the same day (Figure 4A and B).

Intraoperatively, the microscope was used for lesion removal from the neural components in a piecemeal fashion. Preparation for a possible vascular event was made in all cases: the aneurysmal clip and 7/0 nylon sutures were ready to be used in case of VA injury. Identification of the VAs was crucial, as the lesion was very close to the arteries, to ensure adequate resection with total preservation of this structure. In some cases the tumor encased and displaced the adjacent VA posterolaterally; some kinking of the artery as it entered the foramen transversarium was also

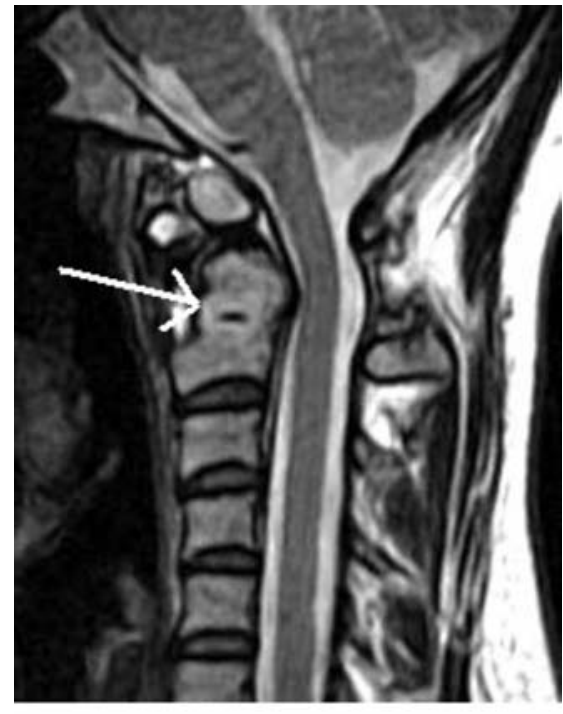

Figure 2. A T2WI sagittal MRI reveals a cystic lesion of the dens with retropulsion of the posterior longitudinal ligament and kyphotic angulation of the cranio-cervical junction - a vivid enhancement after contrast. However, it is still limited to the prevertebral space by the anterior longitudinal ligament (white arrow).

noted. With malignant lesions the tumors appeared destructive to the bony elements, highly vascular with different colors, and fragile lesions. The benign lesions appeared well defined, highly vascular, pink in color, and rubbery lesions. With gentle micro-dissection and piecemeal resection, the tumors were taken out and away from the VAs; none of these tumors invaded the artery. However, some bleeding from the venous plexuses adjacent to the VA was encountered and easily controlled. No intraoperative neural or vascular complications occurred.

All patients had an uneventful postoperative course. Postoperative plain X-rays of the cervical spine were done on all patients; the site of surgical resection and fixation appeared satisfactory, and they started ambulation with a neck collar on the second or third postoperative day. Neurorehabilitation was organized for all cases. Some comorbidities were encountered in some patients: the first case had a back wound with a superficial infection; another patient recovered well from severe sepsis.

\section{Results}

On the regular short- and long-term outpatient assessments of up to four years, no intraoperative neurovascular complications were encountered. Most of the patients displayed a significant neurological recovery in 

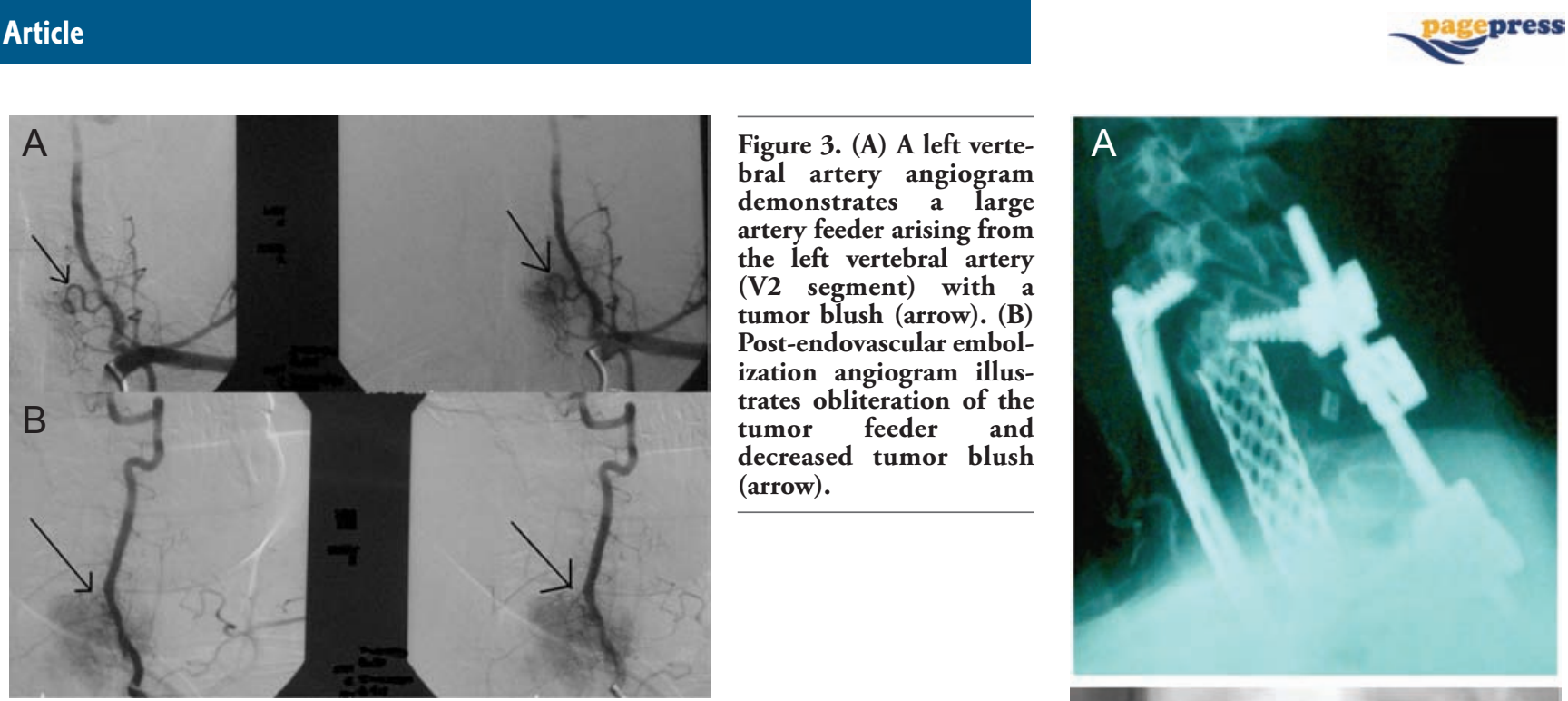

Figure 3. (A) A left vertebral artery angiogram demonstrates a large artery feeder arising from the left vertebral artery (V2 segment) with a tumor blush (arrow). (B) Post-endovascular embolization angiogram illustrates obliteration of the tumor feeder and decreased tumor blush (arrow).

Table 1. Short- and long-term patients' outcomes.

\begin{tabular}{ll}
\hline Motor & One patient continued to have leg weakness. \\
Sensory & Residual numbness along the medial aspect of both arms. \\
\hline Reflexes & Remained increased. \\
Sphincters & Almost back to normal. \\
\hline Gait & Abnormal in one case. \\
Vascular complication & Nil. \\
\hline Mortality & $\begin{array}{l}\text { Three patients died: } \\
\text { one from a massive pulmonary embolism after two months, and } \\
\text { two from local invasion within four months. }\end{array}$ \\
\hline
\end{tabular}

regards motor and sphincteric functions; only two patients continued to have weakness in both legs and needed some aid to become mobile; another two patients continued to have trivial sensory changes along the medial aspect of their arms that required medication. All patients continued to have hyperreflexia. Although three out of the ten patients died, none of them were as a result of the surgery. One was an obese female who had a craniocervical meningioma and was readmitted with respiratory failure two months after her surgery, subsequently passing away from a massive pulmonary embolism. The second case died of local cervical invasion within four months, while the third patient died of malignant metastases (Table 1).

\section{Discussion}

The cervical spine is a common region for trauma and degenerative changes, while neoplastic lesions are relatively rare and usually seen in young age groups. Unlike other parts of the vertebral column, total resection of a cervical spine tumor may not be easy or accessible in all cases because of its closeness and extension to neurovascular structures, particularly the VAs. Patients with cervical spine tumors often present with common symptoms like any other lesion; a long-standing local and/or radicular pain is frequent, and vertebral collapse may compromise the spinal canal and cause radiculo-myelopathy with gait unsteadiness and both sphincter disturbances. Acute symptoms may occur owing to a pathological fracture..$^{6.9}$

The radiological findings of the cervical spine neoplasm are noteworthy. Typically, benign lesions appear well defined with sclerotic edges and are evenly enhancing, whereas malignant lesions appear on plain X-rays and CT scans as expansible, destructive, and radiolucent lesions without sclerotic margins and with soft tissue extensions. MRI findings are more reliable than CT scans..$^{10}$ On T1-weighted images, aggressive lesions may show heterogeneous or homogeneous signals of low intensity. On T2-weighted images, heterogeneous low-to-intermediate signal intensity is seen in solid areas of the tumor. With spinal cord compression, spinal cord signal changes or edema may be evident. Areas of low signal intensity may be exaggerated on T2-weighted images because of hemosiderin deposits, probably as a result of blood leakage. Peritumoral edema is seen with pathological fractures. Spinal deformity in kyphotic angulation may occur owing to vertebral body collapse. VAs may also be encased in or distorted by these lesions. ${ }^{11,12}$

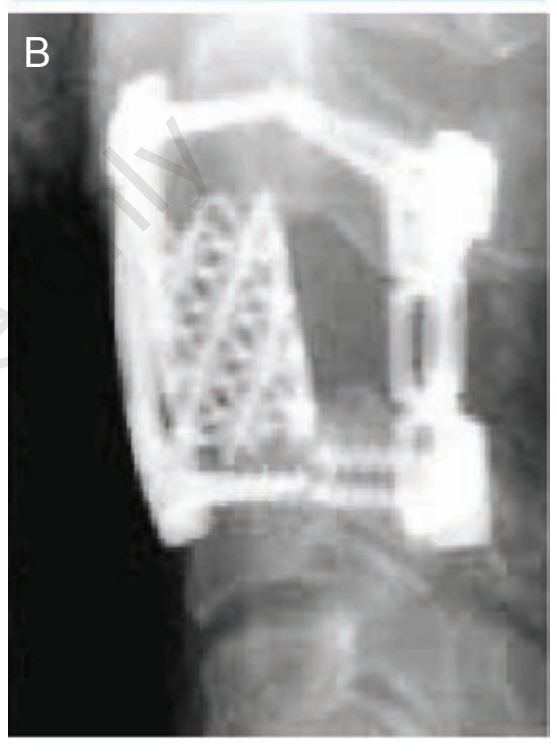

Figure 4. A staged anterior (A) and posterior (B) spinal fixation after corpectomy and total resection of the spinal tumor from front and back.

Preoperative VA angiograms or CT scan angiograms to demonstrate the VAs in relation to the cervical spine and tumor are crucial for planning the surgical technique; the angiogram may help in preoperative endovascular embolization as adjuvant to the surgical treatment. ${ }^{13-15}$ Direct exposure of the artery in pathologically diseased bone is vital to avoid arterial injury, and this can be achieved by a thorough evaluation of the anatomical relationships of the VA, the vertebral body, and the lesion. ${ }^{16}$

Nourbakhsh et al., in their study, illustrated a surgical safe zone for each vertebral body where the surgeon can preserve the VA during the anterior approach and removal of the transverse process. ${ }^{17}$ They concluded that generally the VA is located within $1 \mathrm{~mm}$ above and $2 \mathrm{~mm}$ below the upper vertebral body border, where it is safe to approach the VA. However, 
this could be applicable when the integrity of the vertebral body is preserved and the VA is not distorted. Although some authors demonstrated the possibility of sacrificing one of the VAs without having serious complications, particularly in highly vascular malignant tumors, this seems to be risky. ${ }^{18,19}$

Patients harboring non-spondylotic cervical spine lesions are usually young and active with normal life expectancies. Thus, the treatment objective is to remove the underlying pathology completely and to preserve the spinal integrity. The anatomy of the spine and the difficulty of surgical resection require special consideration. However, a multimodal treatment strategy has been adopted for lesions in this location, including surgery, radiotherapy, endovascular embolization, and adjuvant therapy, as indicated. If applicable, radical surgery remains the optimal treatment with more aggressive lesions. Although it carries a good outcome, complete surgical excision of spinal lesions is not always achievable and it is not without a significant morbidity and mortality. Comorbidities are encountered frequently with radical resection while a high failure rate is seen with inadequate treatment., ${ }^{9,20}$

In general, location and extension of the tumor dictate which surgical approach is used. Lesions involving the anterior parts of the spine require an anterior decompression with stabilization, while with lesions that affect the posterior elements, a laminectomy for decompression of the neural structures and resection of the lesion with posterior fusion is performed. However, in certain cases combined anterior and posterior decompression and instrumental stabilization may be required. To attain adequate treatment, lesions involving this part of the spine require staged operations for both removal and stabilization from front and back, with special precautions focused on the VAs and other adjacent structures..$^{2,6,21-23}$ In most cases en bloc resection of cervical tumors is not attainable. This is because of the tendency of these neoplasms to involve important structures. Intralesional radical removal may be an alternative option for particular cases. ${ }^{24}$

Adjuvant radiotherapy and/or chemotherapy remain a matter of debate but are definitely advocated for cases with incomplete excision of lesions when the cause is malignancy. However, the optimal dose of radiation is yet unknown. The risk of spinal cord myelitis and malignant transformation has been reported. The mainstay predictor of a good outcome is the adequacy of tumor resection and controlled recurrence on routine surveillance. , $^{6,12,16,20}$ Outcome improvement and complication rate reduction for these complex operations can be achieved at specialist centers. Radical surgical intervention should be performed at once with the optimal outcome for the patient being the priority. $^{25}$

\section{Conclusions}

Gross surgical resection of cervical spine neoplastic pathology is the optimal treatment and is feasible. This requires wide surgical experience in order to achieve both adequate surgical resection and preservation of the neurovascular components. Attention to the VAs is to be considered when treating lesions in this area that may also require a neck angiogram and endovascular embolization. Adjuvant radio- or chemotherapy is reserved by many authors for cases of residuals or recurrence. Close surveillance is vital even after gross total resection of the tumor because of the propensity for recurrence. With radical surgical treatment and experience, a long-term satisfactory outcome can be obtained.

\section{References}

1. Brown BM, Schwartz RH, Frank E, et al. Preoperative evaluation of cervical radiculopathy and myelopathy by surface-coil MR imaging. Am J Neuroradiol 1988;9:859-66.

2. Dunn EJ, Davidson RI, Desai S. Diagnosis and management of tumours of the cervical spine. In: The Cervical Spine. The Cervical Spine Research Society Editorial Committee, 2nd edn. Philadelphia: Lippincott, 1989, pp 693-722.

3. Junming M, Cheng Y, Dong C, et al. Giant Cell Tumor of the Cervical Spine: A Series of 22 Cases and Outcomes. Clinical Case Series. Spine 2008;33:280-8.

4. George B, Archilli M, Cornelius JF. Bone tumors at the cranio-cervical junction. Surgical management and results from a series of 41 cases. Acta Neurochir 2006;148:741-9.

5. Bruneau M, Cornelius JF, George B. Anterolateral approach to the V2 segment of the vertebral artery. Neurosurgery 2005;57: S262-7.

6. American Association of Neurological Surgeons/Congress of Neurological Surgeons Joint Section on Disorders of the Spine and Peripheral Nerves. Management of vertebral artery injuries after non-penetrating cervical trauma. Neurosurgery 2002;50:S173-8.

7. Erdogan B, Aydin MV, Sen 0, et al. Giant cell tumour of the sixth cervical vertebrae with close relationship to the vertebral artery. J Clin Neurosci 2005;12:83-5.

8. Hart RA, Boriani S, Biagini R, et al. A system for surgical staging and management of spine tumors. A clinical outcome study of giant cell tumors of the spine. Spine 1997;22:1773-82.

9. Tarantino R, Piccirilli M, Anichini G, Delfini R. Benign osteoblastoma of the odontoid process of the axis with secondary aneurysmal bone cyst component: a case report. Neurosurg Rev 2008;31:111-5.
10. Nakamura M, Toyama Y, Susuki N, et al. Metastases to upper cervical spine. J Spinal Disord 1996;9:195-201.

11. Mastuda Y, Miyazaki K, Tada K, et al. Increased MR signal intensity due to cervical myelopathy: Analysis of 29 cases. J Neurosurg 1991;74:88792.

12. Di Lorenzo N, Spallone A, Nolletti A, et al. Giant cell tumors of the spine: a clinical study of six cases, with emphasis on the radiological features, treatment, and follow-up. Neurosurgery 1980;6:29-34.

13. Trübenbach J, Nägele $\mathrm{T}$, Bauer $\mathrm{T}$, et al. Preoperative embolization of cervical spine osteoblastomas: report of three cases. Am J Neuroradiol 2006;27:1910-2.

14. Rodrigues LM, Nicolau RJ, Puertas EB, et al. Vertebrectomy of giant cell tumor with vertebral artery embolization: case report. J Pediatr Orthop B 2009;18:99-102.

15. Barker R, Fareedi S, Thompson D, et al. The use of CT angiography in the preoperative planning of cervical spine surgery in children. Childs Nerv Syst 2009;25:955-9.

16. Smith MD, Emery SE, Dudley A, et al. Vertebral artery injury during anterior decompression of the cervical spine. A retrospective review of ten patients. J Bone Joint Surg 1993;75:410-5.

17. Nourbakhsh A, Yang J, Gallagher S, et al. A safe approach to explore/identify the V(2) segment of the vertebral artery during anterior approaches to cervical spine and/or arterial repairs: anatomical study. J Neurosurg Spine 2010;12:25-32.

18. Zambelli PY, Lechevallier J, Bracq $\mathrm{H}$, et al. Osteoid osteoma or osteoblastoma of the cervical spine in relation to the vertebral artery. Pediatric Orthop 1994;14:788-92.

19. Sen C, Eisenberg M, Casden AM, et al. Management of the vertebral artery in excision of extradural tumors of the cervical spine. Neurosurgery 1995;36:106-15.

20. Goldenberg RR, Campbell CJ, Bonfiglio M. Giant-cell tumor of bone. An analysis of two hundred and eighteen cases. J Bone Joint Surg Am 1970;52:619-64.

21. Epstein N. Diagnosis and surgical management of ossification of the posterior longitudinal ligament. Contemp Neurosurg 1992;14:1-6.

22. Tekkok IH, Acikgoz B, Sagalam A, et al. Vertebral haemangioma symptomatic during pregnancy - report of a case and review of the literature. Neurosurgery 1993;32:302-6.

23. Fox MW, Onofrio BM. The natural history and management of symptomatic and asymptomatic vertebral hemangioma. J Neurosurgery 1993;78:36-45.

24. Barrenechea IJ, Perin NI, Triana A, et al. Surgical management of chordomas of the cervical spine. J Neurosurg Spine 2007;6:398-406.

25. Choi D, Melcher R, Harms J, et al. Outcome of 132 operations in 97 patients with chordomas of the craniocervical junction and upper cervical spine. Neurosurgery 2010;66:59-65. 